\title{
Estudo de equivalência farmacêutica de comprimidos de cloridrato de metformina genéricos e similares
}

\author{
Pharmaceutical equivalence study of generic and similar metformin \\ hydrochloride tablets
}

Recebido em: 06/09/2019 Aceito em: $13 / 04 / 2020$
Thalita Prates da SILVA ${ }^{1}$; Emilly Isabelli dos Santos TEODORO'; Mariana Nascimento de PAULA ${ }^{1}$; João Carlos Palazzo de MELLO ${ }^{1}$; Daniela Cristina de Medeiros ARAÚJO² ${ }^{1}$ Universidade Estadual de Maringá, Laboratório Palafito. Avenida Colombo, 5790. CEP 87020-900. Maringá, PR, Brasil. . ${ }^{2}$ Centro Universitário Ingá - Uningá. Rod. PR 317, 6114 Parque Industrial 200, CEP 87035-510. Maringá, PR, Brasil. E-mail:danielamedeiros@hotmail.com

\section{ABSTRACT}

Metformin is an oral hypoglycemic of the class of biguanides widely used in the treatment of type 2 diabetes mellitus. Pharmaceutical equivalence studies are aimed at evaluating some quality parameters of medicinal products by comparative analysis between the test drug (s) and the reference dosage form. Thus, the purpose of this study was to evaluate the pharmaceutical equivalence of two brands of generic drug and two brands of similar drug against the reference metformin hydrochloride tablets $850 \mathrm{mg}$. The following tests were carried out: weight variation, friability, disintegration, assay and dissolution. The tested samples were approved in all the tests, meeting the criteria recommended by the Brazilian Pharmacopoeia $5^{\text {a }}$ edition. Thereby, it has been found that all drugs tested are pharmaceutically equivalent to the reference product.

Keywords: metformin; quality control; equivalence trial; drugs, generic; similar drugs.

\section{RESUMO}

A metformina é um hipoglicemiante oral da classe das biguanidas muito utilizado no tratamento de diabetes mellitus tipo 2. Os estudos de equivalência farmacêutica destinam-se à avaliação de alguns parâmetros de qualidade dos medicamentos por meio de análise comparativa entre o(s) medicamento(s) teste e o medicamento de referência. Dessa maneira, o presente trabalho teve como objetivo avaliar a equivalência farmacêutica de comprimidos de duas marcas de medicamento genérico e duas marcas de similar frente ao medicamento referência de cloridrato de metformina $850 \mathrm{mg}$. Os testes realizados nas amostras foram peso médio, teor de substância ativa, friabilidade, desintegração e dissolução. As amostras testadas foram aprovadas em todos os testes, encontrando-se dentro dos limites preconizados pela farmacopeia brasileira $5^{\mathrm{a}}$ edição. Assim pode-se afirmar que todos os medicamentos testados são equivalentes farmacêuticos do medicamento referência.

Palavras-chave: metformina; controle da qualidade; estudo de equivalência; medicamento genérico; medicamento similar. 


\section{INTRODUÇÃO}

A metformina é o hipoglicemiante oral da classe das biguanidas mais empregado no tratamento de diabetes miellitus do tipo 2 , se diferenciando de outros hipoglicemiantes devido a poucos efeitos colaterais, maior segurança e por não promover aumento de peso, podendo até reduzí-lo $(1,2)$. Quando administrada corretamente, tem poucos efeitos adversos, sendo o mais comum o desconforto gastrointestinal como náuseas, indigestão, cólicas e diarreias. Outra reação que pode ocorrer, porém mais rara, é a acidose lática quando em overdose, ou seja, quando há ingestão de metformina muito acima das doses terapêuticas, ou em pessoas com insuficiência hepática, alcoolismo e cirurgias de grande porte $(3,4)$.

Seu mecanismo de ação em relação ao diabetes é ajudar no armazenamento do glicogênio no músculo e aumentar a sensibilidade à insulina, atuando na redução da produção hepática de glicose; reduzindo a absorção de glicose endógena pelo trato gastrointestinal, com pouco risco de hipoglicemia. Além disso, ajuda na manutenção de colesterol LDL e triglicerídeos (1,3-5).

Além dos princípios ativos, os adjuvantes utilizados na formulação também podem interferir na eficácia do medicamento, afetando sua farmacocinética no organismo. Dessa maneira, o controle da qualidade é essencial para que se garanta a ação farmacológica, a eficácia e segurança do medicamento (5).

De acordo com a Resolução de Diretoria Colegiada (RDC) 17/2010 que dispõe sobre Boas Práticas de Fabricação de Medicamentos, o controle da qualidade é o conjunto de técnicas e atividades operacionais utilizadas para monitorar o cumprimento dos requisitos da qualidade especificados (6).

Diretamente relacionado ao controle deaqualidade de medicamentos tem-se a equivalência farmacêutica. Os estudos de equivalência farmacêutica destinam-se à avaliação de alguns parâmetros de qualidade dos medicamentos por meio de análise comparativa entre o medicamento teste e o medicamento de referência. Assim, a equivalência farmacêutica compara se dois ou mais medicamentos apresentam as mesmas características físico-químicas sob a mesma forma farmacêutica (7-9). A RDC
31/2010 dispõe sobre os requisitos para a realização dos estudos de equivalência farmacêutica e de perfil de dissolução comparativo (10).

O medicamento de referência é o resultado da pesquisa de um fármaco inovador que tenha a sua segurança, qualidade e sua eficácia comprovadas, detendo o registro junto à Agência Nacional de Vigilância Sanitária (Anvisa) (11). E o medicamento teste, pode ser um medicamento genérico ou similar, que passará pelo teste de equivalência farmacêutica ou perfil de dissolução (10).

Os medicamentos similares são medicamentos semelhantes ao de referência que possuem a mesma concentração de ativo, forma farmacêutica e via administração, contendo nome comercial (12). Os medicamentos genéricos podem ser intercambiáveis com o medicamento de referência, porém, nhá necessidade de teste de equivalência farmacêutica $\mathrm{e}$ bioequivalência que comprovem sua igualdade ao de referência (11).

A implementação da Lei Dos Genéricos (11) ajudou o acesso da população mais carente ao medicamento, pois os genéricos são no mínimo $35 \%$ menos onerosos do que o medicamento referência, sem prejuízo na qualidade e segurança. Porém eles compõem somente 33\% das prescrições médicas no Brasil (13).Mesmo com resoluções da Anvisa, como a n ${ }^{\circ} 16 / 2007$ e 17/2007, onde é especificado que tanto os genéricos quanto os similares necessitam de testes de bioequivalência e equivalência farmacêutica para ter o registro do medicamento, a população ainda deixa de adquirí-los devido à falta de informação $(12,14,15)$.

Dessa maneira, o presente trabalho teve como objetivo avaliar a equivalência farmacêutica de comprimidos de duas marcas de medicamento genérico (G1 e G2) e duas marcas de similar (S1 e S2) frente ao medicamento referência $(\mathrm{R})$ de cloridrato de metformina $850 \mathrm{mg}$.

\section{MATERIAL E MÉTODO}

As amostras utilizadas foram adquiridas em uma farmácia comercial da cidade de Maringá, e denominadas R para medicamento referência, G1 e G2 para medicamentos genéricos e S1 e S2 para medicamentos similares. 
Foi utilizado um padrão secundário de cloridrato de metformina (Via Farma) com teor de substância ativa $98,5 \%$, nos testes de dissolução e doseamento. Como reagentes do teste de dissolução, foi utilizado fosfato de potássio dibásico (Êxodo Científica) e fosfato de sódio dibásico (Êxodo Científica).

Os equipamentos utilizados foram balanças analítica (Celtac modelo FA2104N); e semi-analítica (BEL modelo MARK 500) aparelho desintegrador (Nova Ética modelo 301-AC); espectrofotômetro de absorção no UV (oceanoptics modelo UBS 2000+); friabilômetro (Nova Ética Mod. 300); dissolutor (Nova Ética modelo 299); e agitador magnético (Fisatom modelo 752A).

A equivalência foi verificada pelos testes de peso médio, doseamento, friabilidade, desintegração, e dissolução, conforme descrito na Farmacopeia Brasileira $5^{\mathrm{a}}$ ed, volumes 1 e $2(16,17)$.

Determinação do peso médio. A determinação do peso médio foi realizada de acordo com o preconizado pela Farmacopeia Brasileira $(16,17)$. Foram pesados 20 comprimidos de cada amostra em balança analítica, seguida da determinação da variação percentual do peso dos comprimidos em relação à média. O desvio padrão foi calculado pela fórmula [1] e o coeficiente de variação pela fórmula [2].

$$
s=\sqrt{\frac{\sum_{i=l}^{n}\left(x_{i}-\bar{x}\right)^{2}}{n-1}}
$$

[1]

Onde: s é o desvio; $\mathrm{n}$ é a quantidade total dos dados do conjunto; $\mathrm{x}_{\mathrm{i}}$ é um valor qualquer no conjunto de dados não posição $\mathrm{i} ; \bar{x}$ é a média aritmética dos dados do conjunto.

$$
c v=\frac{s}{\bar{x}} \cdot 100
$$

Onde: cv é o coeficiente de variação; s é o desvio; $\bar{x}$ é a média aritmética dos dados do conjunto.

Doseamento. Para determinar a quantidade de substância ativa, foram pulverizados os 20 comprimidos utilizados para a determinação do peso médio das amostras R, G1, G2, S1 e S2. Após preparo das amostras, foi realizada a leitura em espectrofotômetro de absorção no ultravioleta em comprimento de onda de $232 \mathrm{~nm}$ e comparado com um padrão secundário de cloridrato de metformina (Via Farma). Água destilada foi utilizada para ajuste do zero e a quantidade de cloridrato de metformina nos comprimidos foi calculada a partir das leituras obtidas.

Preparo do padrão. $100 \mathrm{mg}$ de cloridrato de metformina foram dissolvidos em $70 \mathrm{~mL}$ de água destilada, e agitado em agitador magnético por $15 \mathrm{~min}$. Após o tempo decorrido, foram realizadas diluições sucessivas até a concentração de $0,001 \%(\mathrm{p} / \mathrm{v})$.

Preparo das amostras. Após pesagem de quantidade de pó equivalente a $100 \mathrm{mg}$ de cloridrato de metformina das amostras R, G1, G2, S1 e S2, foram adicionados $70 \mathrm{~mL}$ de água destilada e homogeneizados em agitador magnético por 15 minutos. Após a homogeneização, a mistura foi transferida para balão de $100 \mathrm{~mL}$ e o volume foi completado com o mesmo solvente e filtrado. Foram realizadas diluições sucessivas até a concentração de $0,001 \%$ $(\mathrm{p} / \mathrm{v})$. O teste foi realizado em triplicata para cada amostra, sendo o teor calculado (\%) por meio da seguinte equação [3].

$$
\left(\frac{\mathrm{PPd} \times \mathrm{AbsA} \times \mathrm{PM}}{\mathrm{AbsPd} \times \mathrm{PA} \times \mathrm{DT}}\right) \times 100
$$

Onde: PPd é a massa do padrão; AbsA é a absorbância da amostra; PM é o peso médio dos comprimidos; AbsPd é a absorbância do padrão; PA é a massa da amostra; DT corresponde à dose declarada do medicamento.

Teste de friabilidade. Para o teste de friabilidade, foram pesados 10 comprimidos de cada marca (R, G1, G2, S1 e S2) em balança semi-analítica. Os comprimidos foram colocados no friabilômetro e submetidos a ação do aparelho por 4 min. a 25 rotações/minuto, totalizando 100 rotações.

Após o teste, os comprimidos íntegros e livres de pó foram novamente pesados e a porcentagem de friabilidade foi calculada conforme preconizado pela Farmacopeia Brasileira $(16,17)$. 
Teste de desintegração. O teste de desintegração foi realizado com 6 comprimidos de cada amostra, segundo os critérios descritos na Farmacopeia Brasileira $(16,17)$, utilizando água destilada em temperatura de $37 \pm 1{ }^{\circ} \mathrm{C}$ como líquido de imersão. Os comprimidos foram submetidos à ação do desintegrador por $30 \mathrm{~min}$. Decorrido este tempo, foi analisado se havia a presença de comprimidos ou núcleos duros não desintegrados nos tubos do desintegrador.

Teste de dissolução. O teste de dissolução foi realizado conforme descrito na Farmacopeia Brasileira $(16,17)$, com adaptações $(18,19)$. Foram utilizados $900 \mathrm{~mL}$ do meio de dissolução (tampão fosfato $\mathrm{pH}$ 6,8), dissolutor, aparelhagem (pás; 100 rpm), tempo (45 min.) e temperatura $\left(37 \pm 0,5^{\circ} \mathrm{C}\right)$. Após decorrido o tempo especificado foi retirado uma alíquota $(10 \mathrm{~mL})$ de cada cuba, que foi filtrada e diluída com água até a concentração teórica de $10 \mu \mathrm{g} / \mathrm{mL}$.

Para determinar a quantidade de fármaco dissolvida no meio de dissolução foi realizada a leitura das amostras diluídas em espectrofotômetro de absorção no ultravioleta, no comprimento de onda de $233 \mathrm{~nm}$, e comparado com a leitura obtida para a solução padrão preparada na mesma concentração das amostras. Água destilada foi utilizada para ajuste do zero. O teste foi realizado em triplicata.

\section{RESULTADOS E DISCUSSÃO}

A equivalência farmacêutica entre dois medicamentos corresponde à comprovação de que ambos contêm o mesmo fármaco, isso é, mesmo sal ou éster da mesma molécula terapeuticamente ativa, mesma concentração, forma farmacêutica e via de administração, podendo ou não conter excipientes idênticos. Os testes de equivalência farmacêutica devem ser realizados simultaneamente no medicamento genérico ou medicamento similar, e no respectivo medicamento referência, e se baseiam na comparação dos resultados obtidos (18).

Peso Médio. Os valores obtidos na determinação de peso médio dos comprimidos de cloridrato de metformina $850 \mathrm{mg}(\mathrm{R}, \mathrm{G} 1, \mathrm{G} 2, \mathrm{~S} 1 \mathrm{e} \mathrm{S} 2)$, bem como o desvio padrão (DP) e coeficiente de variação (CV) estão descritos na Tabela 1.

Tabela 1. Resultado do teste de peso médio das amostras de cloridrato de metformina $850 \mathrm{mg}$.

\begin{tabular}{|l|c|c|c|c|c|}
\hline Testes & R & G 1 & G 2 & S 1 & S 2 \\
\hline Peso médio (g) & 0,8943 & 0,8973 & 0,9755 & 1,0110 & 0,9685 \\
\hline Limite da variação de peso (+5\% e -5\%) & 0,9390 e & 0,9422 e & 1,0243 e & $\begin{array}{c}1,0615 \text { e } \\
0,9604\end{array}$ \\
\hline Maior peso encontrado (\%) & 0,8496 & 0,8524 & 0,9267 & 0,9201 \\
\hline Menor peso encontrado (\%) & 1,14 & 1,22 & 1,43 & 1,39 & 2,02 \\
\hline Desvio padrão & 1,21 & 1,20 & 1,31 & 1,36 & 9,72 \\
\hline C. V.(\%) & 0,0058 & 0,0064 & 0,0070 & 0,0064 & 0,0239 \\
\hline Desfecho & 0,6457 & 0,7126 & 0,7125 & 0,6302 & 2,4677 \\
\hline
\end{tabular}

n= 20; C.V.: coeficiente de variação; R: referência; G1: genérico 1; G2: genérico 2; S1: similar 1; S2: similar 2.

Os resultados obtidos na determinação do peso médio das amostras testadas indicaram homogeneidade nos lotes analisados. Segundo a Farmacopeia Brasileira, a variação de peso aceitável para cada comprimido com peso médio de $250 \mathrm{mg}$ ou mais é de $\pm 5 \%$, não sendo permitido mais que duas unida- des fora dos limites especificados e nenhuma unidade acima ou abaixo do dobro desta porcentagem. Foi obtida variação de peso inferior a $5 \%$ na amostra S2, porém não ultrapassou o dobro do limite de $10 \%$. Sendo assim, todas as amostras foram aprovadas neste teste, conforme demonstrado na Tabela 1. 
O peso médio é importante para determinar se os comprimidos formulados possuem o mesmo volume, ou volumes muito próximos entre si, de princípio ativo e adjuvantes farmacêuticos. Este teste é uma ferramenta importante para o controle físico-químico de medicamentos e indica homogeneidade por unidade do lote analisado, podendo indicar problemas no processo de produção, que podem interferir na eficácia terapêutica do medicamento (18).

Doseamento. Os resultados do doseamento foram dispostos na Tabela 2.

Segundo a Farmacopeia Brasileira, a quantidade de cloridrato de metformina presente no comprimido pode variar de $95 \%$ a $105 \%$ do valor declarado no rótulo. Desta forma, todas as amostras analisadas foram aprovadas neste parâmetro de qualidade, variando de $95,5 \%$ a $99,9 \%$, e sendo equivalentes ao medicamento referência em relação ao teor de substância ativa.

Resultados semelhantes foram obtidos por Peres (2013) que realizou estudo de equivalência farmacêutica de comprimidos de cloridrato de metformina $850 \mathrm{mg}$ referência, genérico e similar (9). Os valores de teor obtidos no trabalho de Peres variaram entre $99,71 \%$ e $98,36 \%$, estando dentro dos limites preconizados pela Farmacopeia Brasileira (17).

Tabela 2. Valores obtidos no doseamento dos comprimidos de cloridrato de metformina $850 \mathrm{mg}$ através de espectrofotometria no ultravioleta em $232 \mathrm{~nm}$.

\begin{tabular}{|c|c|c|}
\hline Amostras & Média das massas mg & Teor \% \\
\hline R & $812 \pm 2,8$ & 95,5 \\
\hline G1 & $849 \pm 4,5$ & 99,9 \\
\hline G2 & $812 \pm 2,2$ & 95,5 \\
\hline S1 & $843 \pm 6,0$ & 99,2 \\
\hline S2 & $822 \pm 2,3$ & 96,7 \\
\hline
\end{tabular}

$\mathrm{n}=3$; R: referência; G1: genérico 1; G2: genérico 2; S1: similar 1; S2: similar 2.

O doseamento da substância ativa é importante para determinar a quantidade de substância ativa presente no comprimido, pois a concentração da substância abaixo da especificada pode comprometer a farmacoterapia, e acima pode ocorrer uma superdosagem e até uma overdose, em concentrações muito altas (19).
Teste de friabilidade. Esse teste é aplicável principalmente a comprimidos não revestidos, porém as amostras $\mathrm{R}$ e $\mathrm{G} 1$ que são revestidos foram avaliadas para comparação com as demais. Os valores obtidos neste teste podem ser observados na Tabela 3.

Todos os comprimidos analisados cumpriram com os requisitos do teste, sendo aprovados neste parâmetro de qualidade. O máximo de perda observado nas amostras foi de $0,367 \%(\mathrm{G} 2)$, sendo que nenhum comprimido apresentou-se lascado ou com rachaduras ao final do teste quando observado a olho nu, demonstrando alta resistência a abrasão. As amostras que apresentaram menor perda de massa foram R e G1, possivelmente devido a proteção promovida pelo revestimento.

Tabela 3. Resultados obtidos no teste de friabilidade para os comprimidos de cloridrato de metformina $850 \mathrm{mg}$, referência, genéricos e similares.

\begin{tabular}{|l|c|c|c|c|c|}
\hline Amostras & R & G1 & G2 & S1 & S2 \\
\hline $\begin{array}{l}\text { Peso } \\
\text { Inicial (g) }\end{array}$ & 8,973 & 8,990 & 9,803 & 10,033 & 9,698 \\
\hline Perda (\%) & 0,134 & 0,067 & 0,367 & 0,239 & 0,340 \\
\hline
\end{tabular}

R: referência; G1: genérico 1; G2: genérico 2; S1: similar 1; S2: similar 2.

$\mathrm{Na}$ avaliação da equivalência farmacêutica de comprimidos de furosemida $40 \mathrm{mg}$ similares e genéricos os valores de friabilidade obtidos variaram entre $0,3 \%$ e $0,1 \%$ (20).

Em estudo de avaliação da qualidade e perfil de dissolução de comprimidos de cloridrato de propranolol $40 \mathrm{mg}$ os valores obtidos no teste de friabilidade não ultrapassaram $0,5 \%$ (19).

$\mathrm{Na}$ avaliação de equivalência farmacêutica e estudo comparativo dos perfis de dissolução de medicamentos genéricos contendo paracetamol, o máximo de perda observada nas amostras foi de $0,39 \%$, sendo que nenhum dos comprimidos apresentou-se quebrado, lascado, rachado ou partido ao final do teste (18).

$\mathrm{O}$ teste de friabilidade permite determinar a resistência dos comprimidos à abrasão, quando submetidos à ação mecânica de aparelhagem espe- 
cífica (18). Neste teste, o limite máximo de perda de massa permitido é de $1,5 \%$. Se o resultado for duvidoso ou se a perda for superior ao limite especificado, deve-se repetir o teste por mais duas vezes, considerando, na avaliação, o resultado médio das três determinações (16).

Teste de desintegração. $O$ teste de desintegração permite verificar se a forma farmacêutica se desintegra dentro do limite de tempo especificado, quando seis unidades do lote são submetidas à ação de aparelhagem específica sob condições experimentais descritas. De acordo com a Farmacopeia Brasileira, o tempo máximo permitido para desintegração de comprimidos de cloridrato de metformina é $30 \mathrm{~min}$. Todos os comprimidos testados desintegraram antes do tempo limite, sendo aprovados neste teste (16).

No estudo de equivalência farmacêutica entre comprimidos de dipirona referência, genéricos e similares todos os comprimidos avaliados desintegraram em menos de $30 \mathrm{~min}$, demonstrando equivalência farmacêutica neste parâmetro de qualidade (21).

Em um estudo de equivalência farmacêutica e perfil de dissolução comparativo de comprimidos contendo hidroclorotiazida o tempo máximo de desintegração dos comprimidos testados foi de $15 \min (22)$.

Em estudo comparativo das propriedades físico-químicas de comprimidos de cloridrato de metformina, foi avaliado a desintegração de comprimidos de metformina de $1 \mathrm{~g}$, e todos os comprimidos analisados desintegraram em tempo inferior a $30 \min (5)$.

A avaliação da desintegração dos comprimidos é importante, uma vez que para sua absorção, o fármaco precisa estar disponível para a dissolução (23).

A variação no tempo de desintegração está relacionada com os excipientes utilizados e suas proporções, como por exemplo, excipientes lubrificantes que possuem características hidrofóbicas, assim quanto maior sua quantidade na formulação ocorre o atraso na desintegração e consequentemente na dissolução $(19,22,24,25)$.

Teste de dissolução. Os valores obtidos no teste de dissolução dos comprimidos de cloridrato de metformina 850 mg estão descritos na Tabela 4.
Tabela 4. Resultados obtidos no teste de dissolução dos comprimidos de cloridrato de metformina $850 \mathrm{mg}$.

\begin{tabular}{|c|c|c|}
\hline Amostras & $\begin{array}{l}\text { Substância ativa } \\
\text { dissolvida }(\%)\end{array}$ & $($ Média $\pm \mathrm{DP})$ \\
\hline R 1 & 93,87 & \multirow{3}{*}{$94,14 \pm 0,72$} \\
\hline $\mathrm{R} 1^{\prime}$ & 94,96 & \\
\hline $\mathrm{R} 1^{\prime \prime}$ & 93,60 & \\
\hline G 1 & 86,92 & \multirow{3}{*}{$90,55 \pm 3,15$} \\
\hline $\mathrm{G} 1^{\prime}$ & 92,37 & \\
\hline G $1^{\prime \prime}$ & 92,37 & \\
\hline G 2 & 86,78 & \multirow{3}{*}{$91,46 \pm 4,08$} \\
\hline$G 2^{\prime}$ & 93,32 & \\
\hline G 2" & 94,28 & \\
\hline S 1 & 87,87 & \multirow{3}{*}{$87,28 \pm 4,19$} \\
\hline $\mathrm{S} 1^{\prime}$ & 91,14 & \\
\hline$S 1^{\prime \prime}$ & 82,83 & \\
\hline S 2 & 93,05 & \multirow{3}{*}{$91,73 \pm 8,32$} \\
\hline$S 2^{\prime}$ & 99,32 & \\
\hline S $2^{\prime \prime}$ & 82,83 & \\
\hline
\end{tabular}

R: referência; G1: genérico 1; G2: genérico 2; S1: similar 1; S2: similar 2.

A Farmacopeia Brasileira $(16,17)$ estabelece que para comprimidos de cloridrato de metformina, o valor de Q (porcentagem da quantidade declarada de cloridrato de metformina dissolvida) é $75 \%$, e o tempo do teste é 45 min. Para ser aprovada no estágio 1 (E1) do teste de dissolução, todas as amostras devem apresentar porcentagem de dissolução igual ou superior a $\mathrm{Q}+5 \%(80 \%)$.

De acordo com os valores obtidos, as amostras encontravam-se em conformidade com as especificações estabelecidas, pois apresentaram, individualmente, mais de $80 \%$ do fármaco dissolvido em 45 min (Tabela 4). Porém, é possível perceber uma menor variação entre os comprimidos do medicamento referência, com uma porcentagem de fármaco dissolvido mais homogênea. Na triplicata das amostras de medicamento genérico e similar, foi observada uma maior variação, confirmada pelo maior valor de desvio padrão e coeficiente de variação obtidos.

A dissolução está relacionada com a biodisponibilidade do fármaco; sendo assim, as diferen- 
ças individuais na porcentagem dissolvida entre as amostras testadas de medicamentos genéricos e similares podem acarretar diferenças na sua disponibilidade no organismo (26).

Em um estudo comparativo das propriedades físico-químicas de comprimidos de cloridrato de metformina de $1 \mathrm{~g}$, os valores obtidos de porcentagem dissolvida variaram de $96,32 \%$ a $92,27 \%$. A dissolução do fármaco pode ser influenciada pelos excipientes utilizados na formulação, que podem interferir na desintegração e consequentemente na dissolução $(19,22,24)$.

Block e cols (2008) desenvolveram quatro formulações de comprimidos de metformina 500 $\mathrm{mg}$ com diferentes excipientes e testaram a equivalência dos comprimidos produzidos com o medicamento referência. Os autores observaram que a formulação contendo PVP K30® (polímero não iônico) apresentou menor eficiência de dissolução quando comparada com as outras formulações, incluindo o medicamento referência (27).

O teste de dissolução é utilizado para determinar a quantidade de substância ativa dissolvida em um meio de dissolução (16). Dentre os testes de controle da qualidade físico-química aplicáveis a comprimidos, este teste tem fundamental importância, visto que a extensão da absorção do fármaco depende diretamente da velocidade com que o processo de dissolução ocorre, estabelecendo uma relação direta entre a quantidade dissolvida e a eficácia clínica do medicamento (18).

\section{CONCLUSÃO}

De acordo com os testes realizados, todas as amostras foram aprovadas em relação às análises farmacopeicas de determinação de peso, doseamento, friabilidade e desintegração, confirmando que as amostras de cloridrato de metformina 850 mg analisadas estão aptas a estarem no mercado farmacêutico.

As amostras G1, G2, S1 e S2 apresentaram, no teste de dissolução, uma grande variação de teor de substância ativa dissolvida, verificado pelo desvio padrão e coeficiente de variação das análises. Mesmo que essas variações estejam dentro dos limites preconizados pela Farmacopeia Brasileira, elas indicam uma possível diferença no tempo de ação destes medicamentos quando comparados com o de referência.

Desta forma pode-se inferir que, em relação os testes realizados, as amostras de similares e genéricos testadas são equivalentes farmacêuticos com o medicamento de referência de cloridrato de metformina.

\section{AGRADECIMENTO}

Os autores agradecem ao Laboratório Palafito da Universidade Estadual de Maringá pelo suporte técnico disponibilizado na execução deste trabalho, e ao Centro Universitário Ingá - UNINGÁ.

\section{REFERÊNCIAS}

1. Brunton LL, Chabner BA, Knollmann BC. As bases farmacológicas da terapêutica de Goodman \& Gilman. 12. ed. São Paulo: AMGH, 2012. p.2112.

2. Alves RDC, Nogueira PDS, Barbosa MLC. Fármacos para o tratamento do diabetes tipo ii: uma visita ao passado e um olhar para o futuro. RVq. 2017;9(2):514-534. DOI: $10.21577 / 1984-6835.20170030$

3. Ferreira CBND, Cesaretti MLR, Ginoza M, Kohlmann Jr O. Efeitos da administração de metformina sobre a pressão arterial e o metabolismo glicídico de ratos espontaneamente hipertensos tornados obesos pela inje- ção neonatal de glutamato monossódico. Arq Bras Endocrinol Metabol. 2009;53(4):409-415. DOI: 10.1590/ S0004-27302009000400004

4. Ramos AS, Florencio A, Aldib R, Faria LG. Cloridrato de metformina e as diferenças entre referencial, genérico e similar. In: 2. Simpósio Assistência Farmacêutica 2014. Centro Universitário São Camilo, 2014.

5. Rodrigues TJ, Álvares ACM. Estudo comparativo das propriedades físico- químicas de comprimidos de cloridrato de metformina comercializados no interior de Goiás. REVISA. 2017;6(2):126-133. 
6. BRASIL. Dispõe sobre as Boas Práticas de Fabricação de Medicamentos. Resolução de Diretoria Colegiada (RDC) $N^{0} 301$, de 21 de agosto de 2019. Agência Nacional de Vigilância Sanitária.

7. Storpirtis S, Marcolongo R, Gasparotto FS, Vilanova CMA. Equivalência farmacêutica no contexto da intercambialidade entre medicamentos genéricos e de referência: bases técnicas e científicas. Infarma 2004; 16(9-10):51-56.

8. Pugens AM, Donaduzzi CM, Melo EB. Controle de qualidade total e equivalência farmacêutica de três apresentações de captopril. Rev.Eletr Farm. 2008;5(1):32-45. DOI: $10.5216 /$ ref.v5i1.4612

9. Peres FG. Validação de metodologia analítica para cápsulas magistrais e estudo de equivalência farmacêutica do cloridrato de metformina $850 \mathrm{mg}$ referência, genérico e similar [Dissertação].Brasília: Faculdade de Ciências da Saúde, UnB. 2013.

10. BRASIL. Ministério da Saúde. Dispõe sobre a realização dos Estudos de Equivalência Farmacêutica e de Perfil de Dissolução Comparativo. Agência Nacional de Vigilância Sanitária. Resolução de Diretoria Colegiada (RDC) No 31, de 11 de agosto de 2010. Diário Oficial da União, Brasília, DF, 12 ago. 2010.

11. BRASIL. Ministério da Saúde. Altera a Lei no 6.360, de 23 de setembro de 1976, que dispõe sobre a vigilância sanitária, estabelece o medicamento genérico, dispõe sobre a utilização de nomes genéricos em produtos farmacêuticos e dá outras providências. Agência Nacional de Vigilância Sanitária. Lei No 9.787, de 10 de fevereiro de 1999. Diário Oficial da República Federativa do Brasil, Poder Executivo, Brasília, DF, 10 fev. 1999.

12. BRASIL. Ministério da Saúde. Dispõe sobre o registro de Medicamento Similar e dá outras providências. Agência Nacional de Vigilância Sanitária. Resolução de Diretoria Colegiada (RDC) №. 17, de 02 de Março de 2007. Diário Oficial da União, Brasília, DF, 5 mar. 2007.

13. ABIMG. Progenérico. Associação Brasileira De Indústrias De Medicamentos Genéricos. Mercado. Disponível em: <http://www.progenericos.org.br/index.php/ mercado $>$. Acesso em: 01 maio de 2018.

14. BRASIL. Ministério da Saúde. Aprova Regulamento Técnico para Medicamentos Genéricos. Agência Nacional de Vigilância Sanitária. Resolução de Diretoria Colegiada (RDC) Nº. 16, de 02 de Março de 2007. Diário Oficial da União, Brasília, DF, 5 mar. 2007.

15. Dias JC, Paula Junior W. Percepções e utilização de medicamentos genéricos, similares e referência por pacientes atendidos na unidade básica de saúde do bairro major prates no município de Montes Claros - MG. Rev Farm Fac Santo Agostinho. 2015;5(1):29-45.
16. BRASIL. Farmacopeia Brasileira 5 ed. Ministério da Saúde. Agência Nacional de Vigilância Sanitária. Brasília: Fundação Oswaldo Cruz, 2010. v. 1

17. BRASIL. Farmacopeia Brasileira 5 ed.Ministério da Saúde. Agência Nacional de Vigilância Sanitária. Brasília: Fundação Oswaldo Cruz, 2010. v. 2

18. Brum TF, Laporta LV, Pons Júnior FR, Gonçalves CA, dos Santos MR. Equivalência farmacêutica e estudo comparativo dos perfis de dissolução de medicamentos genéricos contendo paracetamol. Rev. Ciênc. Farm. Básica Apl. 2012;33(3):373-378.

19. Rigobello C, Gasparetto AV, Diniz A, Rabito MF, Nery MMF. Avaliação da qualidade e perfil de dissolução de comprimidos de cloridrato de propranolol. Acta Sci. Health Sci. 2013;35(1):85-90. DOI: 10.4025/actascihealth sci.v35i1.12307

20. Lamolha MA., Rodrigues ACP, Silva BC, Granata FC, Podavin GS, Lima JCO. Avaliação da equivalência farmacêutica de furosemida em comprimidos de 40mg. Rev Bras Farm. 2012;93(1):17-21.

21. Köhler LF, Nascimento HD, Schwengber ELL, Bandeira ZMP, Panzin GV, Machado SRP. Avaliação biofarmacotécnica e perfil de dissolução de comprimidos de dipirona: equivalências farmacêutica entre medicamentos de referência, genéricos e similares. Rev Bras Farm. 2009;90(4):309-315.

22. Ribeiro CC, Andrade GMM, Couto RO. Estudos de equivalência farmacêutica e perfil de dissolução comparativo de medicamentos contendo hidroclorotiazida. Infarma Cien Farm. 2018;30(1):5-13. DOI: 10.14450/2318-9312. v30.e1.a2018.pp5-13

23. Allen LV, Popovich NG, Ansel H. Ansel's Pharmaceutical Dosage forms and drug delivery systems. J. Chem. Inf. Model. 2013. p.1689-1699.

24. Correia LF, Gouvêa MM, Macedo EV, Peregrino CAF, Mourão SC. Avaliação da equivalência farmacêutica de comprimidos de hidroclorotiazida disponíveis no mercado do Rio de Janeiro. Rev. Bras. Farm. 2015;96(2): 1266-1284.

25. Ribeiro RA. Comparação entre diferentes meios de dissolução para comprimidos contendo carvedilol [TCC]. Juiz de Fora: Faculdade de Farmácia, Universidade federal de Juiz de Fora. 2016.

26. Linsbinski LM, Musis CR, Machado SRP, Avaliação da equivalência farmacêutica de comprimidos de captopril. Rev. Bras. Farm. 2008;89(3):214-219.

27. Block LC, Schemling LO, Couto AG, Mourão SC, Bresolin TMB. Pharmaceutical equivalence of metformin tablets with various binders. Rev. Ciênc. Farm. Básica Apl. 2008;29(1):29-35. 